\title{
FUGLEDE-PUTNAM THEOREM AND QUASI-NILPOTENT PART OF $n$-POWER NORMAL OPERATORS
}

\author{
J. STELLA IRENE MARY AND P. VIJAYALAKSHMI
}

\begin{abstract}
In this article we show that the following properties hold for $n$-power normal operators $T$ :

(i) $T$ has the Bishop's property $(\beta)$.

(ii) $T$ is isoloid.

(iii) $T$ is invariant under tensor product.

(iv) $T$ satisfies the Fuglede-Putnam theorem.

(v) $T$ is of finite ascent and descent.

(vi) The Quasi-nilpotent part of $T$ reduces $T$.
\end{abstract}

\section{Introduction}

In this introductory section, we indicate the main trend of the ideas to be developed in this paper. Let $H$ and $K$ be complex Hilbert spaces and $T$ a bounded linear operator on $H$, whose domain, range and null space lie in $H$. Let $L(H)$ denote the algebra of all bounded linear operators acting on $H$. An operator $T$ is said to be $n$-power normal if $T^{*} T^{n}=T^{n} T^{*}$ where $n \in \mathbb{N}$. The class of $n$-power normal operators is denoted by $[n N]$. The class $[n N]$ was introduced by A. S. Jibril [15] and he characterized several properties of class $[n N]$. One of the properties frequently used in this paper is that $T \in[n N]$ if and only if $T^{n}$ is normal. The normality of $T^{n}$ enable us to study several properties of class [ $\left.n N\right]$. For example, in section 2 we give matrix representation for $T$ and $\operatorname{prove} \operatorname{property}(\beta)$.

Definition 1.1. An operator $T \in B(H)$ is said to have the property $(\beta)$ at $\lambda \in \mathbb{C}$ if the following assertion holds:-

If $D \subset \mathbb{C}$ is an open neighbourhood of $\lambda$ and if $f_{n}: D \rightarrow H(n=1,2, \ldots)$ are vector valued analytic functions such that $(T-\mu) f_{n}(\mu) \rightarrow 0$ uniformly on every compact subset of $D$, then $f_{n}(\mu) \rightarrow 0$, again uniformly on every compact subset of $D$, for all $\mu \in D$.

Received May 15, 2014, accepted October 21, 2014.

2010 Mathematics Subject Classification. 47B20.

Key words and phrases. Bishop's property $(\beta)$, isoloid, Fuglede-Putnam Theorem, quasi-nilpotent part. Corresponding author: J. Stella Irene Mary. 
$\operatorname{Property}(\beta)$ has been proved for several operators such as hyponormal operators [21], ( $p, k$ )-quasi-hyponormal operators [26], class $A$ operators [5], class $A(k)$ operators [19], paranormal operators [27], *-paranormal operators [7] and $k$-quasi- $M$-hyponormal operators [24].

Definition 1.2. An operator $T \in L(H)$ is said to be isoloid if every isolated point of $\sigma(T)$ is an eigen value of $T$.

Throughout this paper, the range, null space and the closure of the range of a bounded linear operator $T$, are denoted by ran $\mathrm{T}$, ker $\mathrm{T}$ and $[\mathrm{ranT}]$ respectively. For convenience we write $(T-\lambda)$ in the place of $(T-\lambda I)$.

Two important subspaces in local spectral theory are $\chi_{T}(F)$, the glocal spectral subspace and $\chi_{T}(\mathbb{C}-\{\lambda\})$.

Definition 1.3. For $T \in B(H)$ and a closed subset $F$ of $\mathbb{C}$ the glocal spectral subspace $\chi_{T}(F)$ is defined as the set of all $x \in H$ such that there is an analytic $H$-valued function $f: \mathbb{C} \backslash F \rightarrow H$ for which $(T-\lambda) f(\lambda)=x$ for all $\lambda \in \mathbb{C} \backslash F$.

The quasinilpotent part of $(T-\lambda)$ is denoted by $H_{0}(T-\lambda)$ and defined as follows:

\section{Definition 1.4.}

$$
H_{0}(T-\lambda)=\left\{x \in H: \lim _{n \rightarrow \infty}\left\|(T-\lambda)^{n} x\right\|^{\frac{1}{n}}=0\right\} .
$$

Note that the subspace $\chi_{T}(\{\lambda\})$ coincides with the quasinilpotent part of $(T-\lambda)$ while $\chi_{T}(\mathbb{C}-$ $\{0\})$ coincides with the analytic core $K(T)$ defined as the set $K(T-\lambda)$ of all $x \in H$ such that there exists $c>0$ and a sequence $\left\{x_{n}\right\} \in H$ for which $(T-\lambda) x_{1}=x,(T-\lambda) x_{n+1}=x_{n}$ and $\left\|x_{n}\right\| \leq c^{n}\|x\|$ for all $n \in \mathbb{N}$.

Vrbova' [28] introduced the subspace $K(T)$ which is the analytic counter part of the algebraic core $C(T)$. Saphar [25] introduced the subspace $C(T)$ in purely algebraic terms.

Definition 1.5. Let $T$ be a linear operator on $H$. The algebraic core $C(T)$ is defined to be the greatest subspace $M$ of $H$ for which $T(M)=M$.

We note that $T^{n}(M)=M$ for all $n \in \mathbb{N}$.

The class of all upper semi-Fredholm operators is denoted by $\Phi_{+}(H)$ and is defined as,

$$
\Phi_{+}(H)=\{T \in L(H): \alpha(T)<\infty \text { and } T(H) \text { is closed }\}
$$

and the class of all lower semi-Fredholm operators is denoted by $\Phi_{-}(H)$ and is defined as,

$$
\Phi_{-}(H)=\{T \in L(H): \beta(T)<\infty\}
$$


where $\alpha(T)$ and $\beta(T)$ denote the dimension of the kernel of $T$ and the codimension of the range of $T$. The class of all semi-Fredholm operators is denoted by $\Phi_{ \pm}(H)$ and is defined as $\Phi_{ \pm}(H)=\Phi_{+}(H) \cup \Phi_{-}(H)$ and the class of Fredholm operators is denoted by $\Phi(H)$ and is defined as $\Phi(H)=\Phi_{+}(H) \cap \Phi_{-}(H)$.

Recall that the ascent $p(T)$ of an operator $T$ is the smallest non-negative integer $p$ such that $\operatorname{ker} T^{p}=\operatorname{ker} T^{p+1}$ and if such an integer does not exist then we put $p(T)=\infty$. Analogously, descent $q(T)$ of the operator $T$ is the smallest non-negative integer $q$ such that $\operatorname{ran} T^{q}=\operatorname{ran} T^{q+1}$ and if such an integer does not exist then we put $q(T)=\infty$. If $p(T)$ and $q(T)$ are finite then $p(T)=q(T)[12$, Proposition 38.3].

The class of all Weyl operators denoted by $W(H)$ is defined by,

$$
W(H)=\{T \in \Phi(H) \text { : ind } T=0 \text { where ind } T=\alpha(T)-\beta(T)\} .
$$

\section{Main results}

We begin with the matrix representation for $T \in[n N]$.

Lemma $2.1([15]) . T \in[n N]$ if and only if $T^{n}$ is normal.

Lemma 2.2. Suppose $T \in[n N]$ then $\left[\operatorname{ran} T^{n}\right]$ reduces $T$.

Proof. Since $T \in[n N], T^{n} T^{*}=T^{*} T^{n}$. [ran $\left.T^{n}\right]$ is invariant under $T$ is obvious. We shall show that $\left[\operatorname{ran} T^{n}\right]$ is invariant under $T^{*}$. Let $x \in \operatorname{ran} T^{n}$. Then $x=T^{n} y$ for some $y \in H$ and $T^{*} x=T^{*} T^{n} y=T^{n} T^{*} y \in \operatorname{ran} T^{n}$.

Suppose $z$ is a limit point of $\operatorname{ran} T^{n}$, then there is a sequence $\left\{z_{n}\right\}$ in $\operatorname{ran}\left(T^{n}\right)$ such that $z_{n} \rightarrow z$. Since $\left\{z_{n}\right\}$ is a sequence in $\operatorname{ran} T^{n}, z_{n}=T^{n} x_{n}, n=1,2, \ldots, n \in \mathbb{N}, x_{n} \in H . T^{*} z_{n}=$ $T^{*} T^{n} x_{n}=T^{n} T^{*} x_{n} \in \operatorname{ran} T^{n}$.

So $\left\{T^{*} z_{n}\right\}$ is a sequence in $\operatorname{ran} T^{n}$. By the continuity of $T^{*}$, the sequence $\left\{T^{*} z_{n}\right\} \rightarrow T^{*} z \in$ $\left[\operatorname{ran} T^{n}\right]$. Thus $\left[\operatorname{ran} T^{n}\right]$ is invariant under $T^{*}$ and $\left[\operatorname{ran} T^{n}\right]$ reduces $T$.

Theorem 2.3. If $T$ is $n$-power normal then $T$ has the following matrix representation, $T=$ $\left(\begin{array}{cc}T_{1} & 0 \\ 0 & T_{2}\end{array}\right)$ on $H=\left[\mathrm{ranT}^{n}\right] \oplus \operatorname{ker} T^{* n}$ where $T_{1}=\left.T\right|_{\left[\text {ranT }^{n}\right]}$ is also an n-power normal operator and $T_{2}$ is a nilpotent operator with nilpotency $n$. Futhermore $\sigma(T)=\sigma\left(T_{1}\right) \cup\{0\}$.

Proof. By Lemma 2.2, $\left[\operatorname{ranT}^{n}\right]$ reduces $T$. Hence $T$ has the matrix representation, $T=$ $\left(\begin{array}{cc}T_{1} & 0 \\ 0 & T_{2}\end{array}\right)$ on $H=\left[\operatorname{ran} T^{n}\right] \oplus \operatorname{ker} T^{* n}$. Let $P$ be the orthogonal projection onto $\left[\operatorname{ran} T^{n}\right]$. Then 


$$
\begin{aligned}
& \left(\begin{array}{cc}
T_{1} & 0 \\
0 & 0
\end{array}\right)=T P=P T=P T P . \\
& P\left(T^{n} T^{*}\right) P=\left(\begin{array}{cc}
T_{1}^{n} T_{1}^{*} & 0 \\
0 & 0
\end{array}\right) . \\
& \text { Also } P\left(T^{*} T^{n}\right) P=\left(\begin{array}{cc}
T_{1}^{*} T_{1}^{n} & 0 \\
0 & 0
\end{array}\right) .
\end{aligned}
$$

Since $T \in[n N], P\left(T^{n} T^{*}\right) P=P\left(T^{*} T^{n}\right) P$, implying $T_{1}^{n} T_{1}^{*}=T_{1}^{*} T_{1}^{n}$. Hence $T_{1} \in[n N]$.

For any $z=\left(\begin{array}{c}z_{1} \\ z_{2}\end{array}\right) \in H$,

$$
\begin{aligned}
\left\langle T_{2}^{n} z_{2}, z_{2}\right\rangle & =\left\langle T^{n}(I-P) z,(I-P) z\right\rangle \\
& =\left\langle(I-P) z, T^{* n}(I-P) z\right\rangle \\
& =0 .
\end{aligned}
$$

Therefore $T_{2}^{n}=0$. Since $\left[\operatorname{ran} T^{n}\right]$ reduces $T, \sigma(T)=\sigma\left(T_{1}\right) \cup \sigma\left(T_{2}\right)=\sigma\left(T_{1}\right) \cup\{0\}$.

Lemma 2.4. If $T$ is an n-power normal operator and $M$ is a reducing subspace of $T$ then $\left.T\right|_{M}$ is also an n-power normal operator.

Proof. Since $M$ is a reducing subspace of $T$, it has the matrix representation, $T=\left(\begin{array}{cc}T_{1} & 0 \\ 0 & T_{2}\end{array}\right)$ on $H=M \oplus M^{\perp}$. Let $P$ be the orthogonal projection onto $M$. Then $\left(\begin{array}{cc}T_{1} & 0 \\ 0 & 0\end{array}\right)=T P=P T=P T P$. $P\left(T^{n} T^{*}\right) P=\left(\begin{array}{cc}T_{1}^{n} T_{1}^{*} & 0 \\ 0 & 0\end{array}\right) \cdot P\left(T^{*} T^{n}\right) P=\left(\begin{array}{cc}T_{1}^{*} T_{1}^{n} & 0 \\ 0 & 0\end{array}\right)$.

Since $T \in[n N], T_{1}^{n} T_{1}^{*}=T_{1}^{*} T_{1}^{n}$. Therefore $T_{1} \in[n N]$. Hence $\left.T\right|_{M}$ is n-power normal.

Theorem 2.5. If $T \in[n N]$ then $T$ has the $\operatorname{property}(\beta)$.

Proof. Consider an open neighbourhood $D \subset \mathbb{C}$ of $\lambda \in \mathbb{C}$ and $f_{m}(m=1,2, \ldots)$, the vector valued analytic functions on $D$ such that $(T-\mu) f_{m}(\mu) \rightarrow 0$ uniformly on every compact subset of $D$.

Decompose $H$ as $H=\left[\operatorname{ran} T^{n}\right] \oplus \operatorname{ker} T^{* n}$, by Theorem 2.3, $T=\left(\begin{array}{cc}T_{1} & 0 \\ 0 & T_{2}\end{array}\right)$ where $T_{1} \in[n N]$ and $T_{2}$ is a nilpotent operator with nilpotency $n$.

$(T-\mu) f_{m}(\mu) \rightarrow 0$ implies,

$$
\left(\begin{array}{cc}
T_{1}-\mu & 0 \\
0 & T_{2}-\mu
\end{array}\right)\left(\begin{array}{c}
f_{m_{1}}(\mu) \\
f_{m_{2}}(\mu)
\end{array}\right)=\left(\begin{array}{c}
\left(T_{1}-\mu\right) f_{m_{1}}(\mu) \\
\left(T_{2}-\mu\right) f_{m_{2}}(\mu)
\end{array}\right) \rightarrow 0 .
$$

Since $T_{2}$ is nilpotent, it has property $(\beta)$ and therefore $f_{m_{2}}(\mu) \rightarrow 0$. 
Also since $T_{1}^{n}$ is normal, it has $\operatorname{property}(\beta)$ and therefore by Theorem 3.39 [17], $T$ has $\operatorname{property}(\beta)$.

Corollary 2.6. If $T \in[n N]$ then $T$ has the single-valued extension property.

The following two Examples show that for a $n$ - power normal operator $T$, the corresponding eigenspaces need not be reducing subspaces of $T$.

Example 2.7. $T=\left(\begin{array}{ll}0 & 1 \\ 0 & 0\end{array}\right)$. Clearly $T$ is a 2-power normal operator and the eigenspace of $T$ is $\left(\begin{array}{l}x \\ 0\end{array}\right)$ but it is not a reducing subspace of $T$.

Example 2.8. $T=\left(\begin{array}{cc}1 & 2 \\ 0 & -1\end{array}\right)$. Here $T$ is a 2-power normal operator and the corresponding eigenspaces of $T$ are $\left(\begin{array}{l}x \\ 0\end{array}\right)$ and $\left(\begin{array}{c}x \\ -x\end{array}\right)$ but these are not reducing subspaces of $T$.

Also the $n$-power normal operators are not semiregular. For example consider the multiplication operator $T$ defined by $(T f)(t)=t f(t)$ for $f \in L^{2}[0,1]$ and $t \in[0,1]$. Then $T$ is normal, injective and has dense range. Since the range of $T$ is not closed, $T$ is not semiregular.

Lemma 2.9. Let $T \in[n N]$ and $\lambda \in \sigma(T)$ be an isolated point.Then $\lambda^{n}$ is an isolated point of $\sigma\left(T^{n}\right)$.

Proof. Since $\lambda \in \sigma(T)$ is an isolated point there is a neighbourhood $V$ of $\lambda$ with radius $\delta$ which contains no point of $\sigma(T)$ other than $\lambda$. By Spectral mapping Theorem, $\lambda^{n} \in \sigma\left(T^{n}\right)$. Suppose $\lambda^{n} \in \sigma\left(T^{n}\right)$ is not an isolated point of $\sigma\left(T^{n}\right)$, then every neighbourhood of $\lambda^{n}$ contains atleast one point of $\sigma\left(T^{n}\right)$ other than $\lambda^{n}$. Consequently, let $\mu^{n}$ in $\sigma\left(T^{n}\right)$ be a point in a neighbourhood $V_{n}$ of $\lambda^{n}$ with radius $\frac{\delta}{2} \rho$ where $\rho=\left|\sum_{k=0}^{n-1} \lambda^{n-k-1} \mu^{k}\right|$. It follows from Spectral mapping Theorem that $\mu \in \sigma(T)$. Then

$$
\begin{aligned}
\left|\lambda^{n}-\mu^{n}\right| & \leq \frac{\delta}{2} \rho \\
\left|\lambda^{n}-\mu^{n}\right| & =|\lambda-\mu|\left|\sum_{k=0}^{n-1} \lambda^{n-k-1} \mu^{k}\right| \\
& =|\lambda-\mu| \rho \\
\text { Consequently, } \quad|\lambda-\mu| & \leq \frac{\delta}{2} \text { by (2.1). }
\end{aligned}
$$

This shows that $\mu \neq \lambda$ is a point in $V$, contradicting the hypothesis that $\lambda$ is an isolated point of $\sigma(T)$. Thus, $\lambda^{n}$ is an isolated point of $\sigma\left(T^{n}\right)$. 
Lemma 2.10. Let $T \in[n N]$ then $T$ is isoloid.

Proof. Let $\lambda \in \sigma(T)$ be an isolated point. By Lemma 2.9, $\lambda^{n}$ is an isolated point of $\sigma\left(T^{n}\right)$. Since $T^{n}$ is normal, it is isoloid. Therefore $\lambda^{n}$ is in the point spectrum of $T^{n}$. This implies that $\left(\lambda^{n}-T^{n}\right)^{-1}$ does not exist.

$$
\left(\lambda^{n}-T^{n}\right)^{-1}=\frac{1}{\lambda^{n}}\left(I-\frac{T^{n}}{\lambda^{n}}\right)^{-1}=\mu^{n}\left(I-\mu^{n} T^{n}\right)^{-1} \text { where } \mu=\frac{1}{\lambda} .
$$

Recall the identity,

$$
\begin{aligned}
& \left(I-\mu^{n} T^{n}\right)^{-1}=\frac{1}{n}\left[(I-\mu T)^{-1}+(I-\mu w T)^{-1}+\left(I-\mu w^{2} T\right)^{-1}+\cdots+\left(I-\mu w^{n-1} T\right)^{-1}\right] \\
& \left(I-\mu^{n} T^{n}\right)^{-1}=\frac{1}{n}\left[(I-\mu T)^{-1}+\sum_{k=1}^{n-1}\left(I-\mu w^{k} T\right)^{-1}\right]
\end{aligned}
$$

where $w$ is the primitive root of unity. Since $\left(I-\mu^{n} T^{n}\right)^{-1}$ does not exist, atleast one term of the expression on the righthand side of (2.2) does not exist. Hence there exist two cases.

Case(i):

Suppose $(I-\mu T)^{-1}$ does not exist. Since $\mu=\frac{1}{\lambda},(\lambda I-T)^{-1}$ does not exist, which implies $\lambda \in$ $P_{\sigma}(T)$.

\section{Case(ii):}

Suppose $\left(I-\mu w^{k} T\right)^{-1}$ does not exist for some $k=1,2,3, \ldots, n-1$. From (2.2),

$$
\begin{aligned}
n\left(I-\mu^{n} T^{n}\right)^{-1}-\left(I-\mu w^{k} T\right)^{-1}= & (I-\mu T)^{-1}+\cdots+\left(I-\mu w^{k-1} T\right)^{-1} \\
& +\left(I-\mu w^{k+1} T\right)^{-1}+\cdots+\left(I-\mu w^{n-1} T\right)^{-1} .
\end{aligned}
$$

Since $n\left(I-\mu^{n} T^{n}\right)^{-1}-\left(I-\mu w^{k} T\right)^{-1}$ does not exist the expression on the otherside also does not exist. In that expression atleast one term does not exist. On repeating a similar argument as above, we arrive at a stage where $(I-\mu T)^{-1}$ does not exist. That is $\frac{1}{\lambda}(\lambda I-T)^{-1}$ does not exist, hence $\lambda \in P_{\sigma}(T)$. It follows that $T$ is isoloid.

\section{Tensor product of class $[\mathrm{nN}]$ operators}

For $A, B \in L(H)$, a number of authors have considered variously, the tensor product $A \otimes B$, on the product space $H \otimes H$. The operation of taking tensor products $A \otimes B$ preserves many a property of $A, B \in L(H)$, but by no means all of them. For instance the normaloid property is invariant under tensor products, whereas the spectroloid property is not [23, pp.623 and 631]. H. Jinchuan [16] proved that $A \otimes B$ is normal if and only if $A$ and $B$ are so, where $A$ and $B$ are non-zero operators. Similar results were proved for subnormal operators [18], hyponormal operators [13], $p$ - hyponormal operators [6], class $A$ operators [14] and $p$ - quasihyponormal operators [9]. But there exists paranormal operators $A$ and $B$ such that $A \otimes B$ is not paranormal [3]. We show that if $A$ and $B$ are of class n-power normal then $A \otimes B$ is also of the class n-power normal. 
Lemma 2.11 ([13]). If $A \in L(H)$ and $B \in L(K)$ are non-zero operators, then $A \otimes B$ is normal if and only if so are $A$ and $B$.

Theorem 2.12. $T_{1} \otimes T_{2}$ is an n-power normal operator if and only if $T_{1}$ and $T_{2}$ are so.

Proof. First we begin with the observations that $\left(T_{1} \otimes T_{2}\right)^{*}\left(T_{1} \otimes T_{2}\right)=T_{1}^{*} T_{1} \otimes T_{2}^{*} T_{2}$ and $\left(T_{1} \otimes\right.$ $\left.T_{2}\right)^{n}=T_{1}^{n} \otimes T_{2}^{n}$. Suppose $T_{1}$ and $T_{2}$ are n-power normal operators, then

$$
\begin{aligned}
\left(T_{1} \otimes T_{2}\right)^{n}\left(T_{1} \otimes T_{2}\right)^{*} & =T_{1}^{n} T_{1}^{*} \otimes T_{2}^{n} T_{2}^{*} \\
& =T_{1}^{*} T_{1}^{n} \otimes T_{2}^{*} T_{2}^{n} \\
& =\left(T_{1} \otimes T_{2}\right)^{*}\left(T_{1} \otimes T_{2}\right)^{n}
\end{aligned}
$$

Therefore $T_{1} \otimes T_{2}$ is an n-power normal operator. Conversely, suppose $T_{1} \otimes T_{2}$ is an n-power normal operator, then $\left(T_{1} \otimes T_{2}\right)^{n}$ is normal. By Lemma 2.11 , we have $T_{1}^{n}$ and $T_{2}^{n}$ are normal. Then by Lemma 2.1, $T_{1}$ and $T_{2}$ are n-power normal operators.

\section{Fuglede-Putnam Theorem for n-power normal operators}

Fuglede-Putnam Theorem is well known in operator theory. It affirms that if $A$ and $B$ are normal operators and $A X=X B$ for some operator $X$ then $A^{*} X=X B^{*}$. First, Fuglede [10] proved it in the case when $A=B$ and then Putnam [22] proved it in a general case. There exists many generalizations of this Theorem of which most of them go into unwinding the normality of $A$ and $B$ (see $[11,20]$ and some of the references cited in these papers).

Berbarian [4] unwinds the hypothesis on $A$ and $B$ by assuming $A$ and $B^{*}$ are hyponormal operators and $X$ to be a Hilbert-Schmidt class. The operators in $H$ which are of Hilbert-

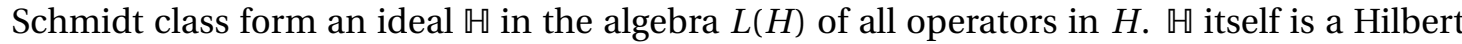
space for the inner product

$$
\langle X, Y\rangle=\sum\left\langle X e_{i}, Y e_{i}\right\rangle=\operatorname{Tr}\left(Y^{*} X\right)=\operatorname{Tr}\left(X Y^{*}\right),
$$

where $\left\{e_{i}\right\}$ is any orthonormal basis of $H$. For each pair of operators $A, B \in L(H)$, there is an operator $\Gamma$ defined on $L(\mathbb{M})$ via the formula $\Gamma(X)=A X B$ as in [4]. Obviously, $\|\Gamma\| \leq\|A\|\|B\|$. The adjoint of $\Gamma$ is given by the formula $\Gamma^{*}(X)=A^{*} X B^{*}$. Also if $A \geq 0, B \geq 0$ then $\Gamma \geq 0$ [4].

Lemma 2.13. If $A$ and $B^{*}$ are of class $[n N]$ then the operator $\Gamma$ is of class $[n N]$.

Proof. By hypothesis, $A^{*} A^{n}=A^{n} A^{*}, B B^{*^{n}}=B^{*^{n}} B$.

Since, $\Gamma(X)=A X B$ and $\Gamma^{*}(X)=A^{*} X B^{*}$ for any pair $A, B \in L(H)$,

$$
\begin{aligned}
\left(\Gamma^{*} \Gamma^{n}-\Gamma^{n} \Gamma^{*}\right) X & =\Gamma^{*} \Gamma^{n} X-\Gamma^{n} \Gamma^{*} X \\
& =\Gamma^{*}\left(A^{n} X B^{*^{n}}\right)-\Gamma^{n}\left(A^{*} X B\right)
\end{aligned}
$$




$$
\begin{aligned}
& =A^{*} A^{n} X B^{*^{n}} B-A^{n} A^{*} X B B^{*^{n}} \\
& =A^{*} A^{n} X B^{*^{n}} B-A^{*} A^{n} X B^{*^{n}} B \\
& =0 .
\end{aligned}
$$

The above equality shows that $\Gamma \in[n N]$.

Lemma 2.14. If $A \in[n N]$ and $A$ is invertible, then $A^{-1} \in[n N]$.

Proof. By hypothesis $A^{*} A^{n}=A^{n} A^{*}$, we need to prove that $A^{*^{-1}} A^{n^{-1}}=A^{n^{-1}} A^{*^{-1}}$.

$$
A^{*^{-1}} A^{n^{-1}}=\left(A^{n} A^{*}\right)^{-1}=\left(A^{*} A^{n}\right)^{-1}=A^{n^{-1}} A^{*^{-1}} .
$$

Hence $A^{-1} \in \operatorname{class}[n N]$.

Lemma 2.15 ([15]). Let $T \in L(H)$ such that $T \in[2 N] \cap[3 N]$, then $T \in[n N]$ for all positive integers $n \geq 4$.

Theorem 2.16. Let $A$ and $B^{*}$ be in class $[2 N \cap 3 N]$ such that $B^{*}$ is invertible and $X$ be a HilbertSchmidt operator. Suppose that $A X=X B$ then $A^{*} X=X B^{*}$.

Proof. Let $\Gamma$ be the Hilbert-Schmidt operator defined by, $\Gamma Y=A Y B^{-1}$, where $Y \in L(H)$. By hypothesis $A$ and $B^{*}$ are of class $[n N]$, by Lemma $2.14\left(B^{*}\right)^{-1}$ is of class $[n N]$. Since $\left(B^{*}\right)^{-1}=$ $\left(B^{-1}\right)^{*}$, it follows by Lemma 2.13 that $\Gamma$ is of class $[n N]$. The hypothesis $A X=X B$ implies that $\Gamma X=X$ and also by Lemma 2.15, $T \in[n N]$ for all $n \geq 2$, it follows that,

$$
\begin{aligned}
\left\|\Gamma^{*} X\right\|^{2} & =\left\langle\Gamma^{*} X, \Gamma^{*} X\right\rangle \\
& =\left\langle\Gamma^{*} \Gamma^{n} X, \Gamma^{*} \Gamma^{n} X\right\rangle \\
& =\left\langle\Gamma \Gamma^{* n} \Gamma^{*} \Gamma^{n} X, X\right\rangle \\
& =\left\langle\Gamma \Gamma^{* n+1} \Gamma^{n} X, X\right\rangle \\
& =\left\langle\Gamma^{* n+1} \Gamma \Gamma^{n} X, X\right\rangle \\
& =\left\langle\Gamma^{n+1} X, \Gamma^{n+1} X\right\rangle \\
& =\|X\|^{2} .
\end{aligned}
$$

The above equality gives,

$$
\begin{aligned}
\left\|\Gamma^{*} X-X\right\|^{2} & =\left\langle\Gamma^{*} X-X, \Gamma^{*} X-X\right\rangle \\
& =\left\langle\Gamma^{*} X, \Gamma^{*} X\right\rangle-\left\langle\Gamma^{*} X, X\right\rangle-\left\langle X, \Gamma^{*} X\right\rangle+\langle X, X\rangle \\
& =\left\|\Gamma^{*} X\right\|^{2}-\langle X, \Gamma X\rangle-\langle\Gamma X, X\rangle+\|X\|^{2} \\
& =\|X\|^{2}-\langle X, X\rangle-\langle X, X\rangle+\|X\|^{2}
\end{aligned}
$$




$$
=0 .
$$

Therefore $\Gamma^{*} X=X$ and hence $A^{*} X=X B^{*}$.

\section{Ascent and Descent}

The non-negative integers $p(T)$ and $q(T)$ known as the ascent and descent of $T$ respectively play a vital role to generate several classes of Browder operators and related spectrum. So we may anticipate if an n-power normal operator $T$ have finite ascent(descent) or not. Infact, $T^{n}$ has finite ascent since it is normal. Indeed, the following Lemma shows that the ascent and descent of $T \in[n N]$ are finite.

Lemma 2.17. For any operator $T \in L(H)$ with $T^{n}$ normal, the following assertions hold:

(i) $p(T)=q(T) \leq n$.

(ii) $N^{\infty}(T)=\operatorname{ker} T^{n}$ and $T^{\infty}(H)=\operatorname{ran} T^{n}$, where $N^{\infty}(T)=\bigcup_{k \in \mathbb{N}} \operatorname{ker} T^{k}$ and

$T^{\infty}(H)=\bigcap_{k \in \mathbb{N}} T^{k}(H)$ are the hyper kernel and hyper range respectively.

Proof. (i) It is well known that, for any normal operator $A, \operatorname{ker} A^{2}=\operatorname{ker} A$ and $\left[\operatorname{ran} A^{2}\right]=$ $[\operatorname{ran} A]$.

Since $T^{n}$ is normal, $\operatorname{ker} T^{2 n}=\operatorname{ker} T^{n}$ and $\left[\operatorname{ran} T^{2 n}\right]=\left[\operatorname{ran} T^{n}\right]$. Consequently, from the chain relations $k e r T \subseteq \operatorname{ker} T^{2} \subseteq \cdots \subseteq \operatorname{ker} T^{n} \subseteq \operatorname{ker} T^{n+1} \subseteq \cdots \subseteq \operatorname{ker} T^{2 n}=\operatorname{ker} T^{n} \subseteq \operatorname{ker} T^{2 n+1} \ldots$ and $\cdots\left[\operatorname{ranT} T^{n}\right]=\left[\operatorname{ranT} T^{2 n}\right] \subseteq\left[\operatorname{ranT} T^{2 n-1}\right] \subseteq \cdots \subseteq\left[\operatorname{ranT} T^{n+1}\right] \subseteq\left[\operatorname{ranT} T^{n}\right] \subseteq\left[\operatorname{ranT} T^{n-1}\right] \subseteq \cdots \subseteq$ $[\operatorname{ran} T]$, we obtain, $\operatorname{ker} T^{n}=\operatorname{ker} T^{n+1}$ and $\operatorname{ran} T^{n}=\operatorname{ran} T^{n+1}$. By the definition of $p(T)$ and $q(T)$, we have $p(T) \leq n$ and $q(T) \leq n$. Since both are finite $p(T)=q(T)$ [12].

(ii) Also

$$
N^{\infty}(T)=\bigcup_{k \in \mathbb{N}} \operatorname{ker} T^{k}=\operatorname{ker} T^{n}, T^{\infty}(H)=\bigcap_{k \in \mathbb{N}} T^{k}(H)=\operatorname{ran} T^{n} .
$$

\section{Nullity and Deficiency}

The role of nullity $\alpha(T)$ and deficiency $\beta(T)$ of an operator $T$ are crucial in the class of Fredholm operators and Weyl operators. The following Theorem concerning $\alpha(T)$ and $\beta(T)$ is useful to explore if $T \in[n N]$ fit into the class of Weyl operators or not. Infact, Aiena [1] proved a Theorem connecting ascent and descent with nullity and deficiency, which is stated below.

Theorem 2.18 ([1],Theorem 3.4). If $T$ is a linear operator on a vector space $X$ and if $p(T)=$ $q(T)<\infty$ then $\alpha(T)=\beta(T)$ (possibly infinity).

Theorem 2.19. Suppose $T \in[n N]$ such that $\alpha(T)$ or $\beta(T)$ is finite and $T(H)$ is closed then $T$ is a Weyl operator. 
Proof. We have $p(T)=q(T) \leq n$ by Lemma 2.17. It immediately follows from Theorem 2.18 that $\alpha(T)=\beta(T)<\infty$.

Consequently $T$ is a Fredholm operator with ind $\mathrm{T}=0$ and hence Weyl.

Theorem 2.20. Suppose that $C(T)$ is the algebraic core of $T \in[n N]$ then the following assertions hold:

(i) $C(T)$ is invariant under $T^{* n}$.

(ii) $T^{*}(C(T)) \subseteq C\left(T^{n}\right)$.

Proof. (i) Since $T \in[n N], T^{*} T^{n}=T^{n} T^{*}$ or $T^{* n} T=T T^{* n}$. Also by the definition of algebraic core of $T, T(C(T))=C(T)$ or $T^{n}(C(T))=C(T)$ for all $n \in \mathbb{N}$. $T^{* n} T=T T^{* n}$ implies $T^{* n} T(C(T))=T T^{* n}(C(T))$ or $T^{* n}(C(T))=T T^{* n}(C(T))$.

$C(T)$ being the greatest subspace satisfying $T(C(T))=C(T)$, we have $T^{* n}(C(T)) \subseteq C(T)$. Thus $C(T)$ is invariant under $T^{* n}$.

(ii) $T \in[n N]$ implies $T^{*} T^{n} C(T)=T^{n} T^{*} C(T)$ or $T^{*}(C(T))=T^{n} T^{*}(C(T))$. It follows that $T^{*}(C(T)) \subseteq C\left(T^{n}\right)$, the algebraic core of $T^{n}$.

A. S. Jibril [15] proved that if $T \in[2 N] \cap[3 N]$, then $T \in[n N]$ for all positive integers $n \geq 4$.

Theorem 2.21. If $T \in[2 N] \cap[3 N]$, then

(i) $H_{0}(T)$ is a reducing subspace of $T$.

(ii) $x \in H_{0}(T)$ if and only if $T^{*} x \in H_{0}(T)$ where

$$
H_{0}(T)=\left\{x \in H: \lim _{n \rightarrow \infty}\left\|T^{n} x\right\|^{\frac{1}{n}}=0\right\} .
$$

(iii) $\operatorname{ker}(T-\lambda) \cap H_{0}(T)=\{0\}$ for every $\lambda \neq 0$.

Proof. (i) Let $F \subset \mathbb{C}$ be a closed set. The glocal spectral subspace $\chi_{T}(F)$ is defined as, $\chi_{T}(F)=$ $\{x \in H: \exists$ analytic $f(z):(T-z) f(z)=x$ on $\mathbb{C} \backslash F\}$. By Theorem 2.20 [1], we have $H_{0}(T-\lambda)=$ $\chi_{T}(\{\lambda\})$. By Theorem 2.5, $T$ has property $(\beta)$. Also by Proposition 1.2.19 [17], $\chi_{T}(F)$ is closed and $\sigma\left(\left.T\right|_{\chi_{T}(F)}\right) \subset F$. Hence $H_{0}(T-\lambda)$ is closed for $\lambda \in \mathbb{C}$, which implies $H_{0}(T)$ is closed. If $x \in H_{0}(T)$ then from the inequality $\left\|T^{n} T x\right\| \leq\|T\|\left\|T^{n} x\right\|$, it is easily seen that $T x \in H_{0}(T)$ and $H_{0}(T)$ is invariant under $T$.

$$
\begin{aligned}
\left\|T^{n} T^{*} x\right\|^{2} & =\left\langle T^{n} T^{*} x, T^{n} T^{*} x\right\rangle \\
& =\left\langle T T^{* n} T^{n} T^{*} x, x\right\rangle \\
& =\left\langle T^{* n+1} x, T^{* n+1} x\right\rangle \\
& =\left\|T^{n+1} x\right\|^{2} \text { since } T^{n+1} \text { is normal } \\
\left\|T^{n} T^{*} x\right\| & =\left\|T^{n+1} x\right\|
\end{aligned}
$$


If $x \in H_{0}(T)$ then, $\left\|T^{n} T^{*} x\right\|^{\frac{1}{n}}=\left(\left\|T^{n+1} x\right\|^{\frac{1}{n+1}}\right)^{\frac{n+1}{n}}$ by (2.3). It follows that $T^{*} x \in H_{0}(T)$ and $H_{0}(T)$ is invariant under $T^{*}$.

(ii) $x \in H_{0}(T)$ implies $T^{*} x \in H_{0}(T)$ follows by (i). Conversely let $T^{*} x \in H_{0}(T)$. Since by (2.3) $\left\|T^{n+1} x\right\|=\left\|T^{n} T^{*} x\right\|$,

$$
\lim _{n \rightarrow \infty}\left\|T^{n+1} x\right\|^{\frac{1}{n+1}}=\lim _{n \rightarrow \infty}\left(\left\|T^{n} T^{*} x\right\|^{\frac{1}{n}}\right)^{\frac{n}{n+1}}=0 .
$$

Thus $x \in H_{0}(T)$.

(iii) Suppose $x \neq 0 \in \operatorname{ker}(T-\lambda) \cap H_{0}(T)$. Then $x \in \operatorname{ker}(T-\lambda)$ implies,

$$
(T-\lambda) x=0 \Rightarrow T x=\lambda x \Rightarrow T^{n} x=\lambda^{n} x .
$$

By (ii) $x \in H_{0}(T)$ if and only if $T^{*}(x) \in H_{0}(T)$ and hence,

$$
\begin{aligned}
0 & =\lim _{n \rightarrow \infty}\left\|T^{n} T^{*} x\right\|^{\frac{1}{n}} \\
& =\lim _{n \rightarrow \infty}\left\|T^{*} T^{n} x\right\|^{\frac{1}{n}} \\
& =\lim _{n \rightarrow \infty}\left\|T^{*} \lambda^{n} x\right\|^{\frac{1}{n}} \\
& =\lim _{n \rightarrow \infty}|\lambda|\left\|T^{*} x\right\|^{\frac{1}{n}} \\
& =|\lambda| \lim _{n \rightarrow \infty}\left\|T^{*} x\right\|^{\frac{1}{n}} \\
& =|\lambda| .
\end{aligned}
$$

Which is a contradiction and therefore $T^{*} x \notin H_{0}(T) \Rightarrow x \notin H_{0}(T)$. Hence $\operatorname{ker}(T-\lambda) \cap H_{0}(T)=$ $\{0\}$ for every $\lambda \neq 0$.

Remark 2.22. For $T \in[n N]$ the restriction $\left.T^{n}\right|_{M}$ of $T^{n}$ to a closed invariant subspace $M$ is a hyponormal operator, since $\left.T^{n}\right|_{M}$ is subnormal.

Theorem 2.23. Suppose $T \in[2 N] \cap[3 N]$, then for every $m \geq 2, m \in \mathbb{N}$ the following properties hold:

(i) $H_{0}\left(T^{m}-\lambda\right)$ is a reducing subspace of $T$.

(ii) $H_{0}\left(T^{m}-\lambda\right)=\operatorname{ker}\left(T^{m}-\lambda\right)=\operatorname{ker}\left(T^{* m}-\lambda\right)$. In particular $H_{0}\left(T^{m}\right)=\operatorname{ker} T^{m}=\operatorname{ker} T^{* m}$.

(iii) If $M$ is an invariant subspace of $T$ and $T_{1}=\left.T\right|_{M}$ on $H=M \oplus M^{\perp}$ then $H_{0}\left(T_{1}^{m}-\lambda\right)=$ $\operatorname{ker}\left(T_{1}^{m}-\lambda\right) \subseteq \operatorname{ker}\left(T_{1}^{m}-\lambda\right)^{*}$.

(iv) $H_{0}\left(T^{m}-\lambda^{m}\right) \supset H_{0}(T-\lambda)$ and $H_{0}\left(T^{m}-\lambda^{m}\right)=H_{0}(T-\lambda)$ if $S=T^{m-1}+\lambda T^{m-2}+\cdots+\lambda^{m-2} T+$ $\lambda^{m-1}$ is invertible.

(v) $H_{0}(T-\lambda) \subset \operatorname{ker}\left(T^{m}-\lambda^{m}\right)$ and $H_{0}(T-\lambda)=\operatorname{ker}(T-\lambda)$ if $S$ is invertible. 
Proof. (i)

$$
H_{0}\left(T^{m}-\lambda\right)=\left\{x \in H: \lim _{n \rightarrow \infty}\left\|\left(T^{m}-\lambda\right)^{n} x\right\|^{\frac{1}{n}}=0\right\}
$$

Since $T \in[2 N] \cap[3 N], T$ is n-power normal for all $n \geq 2$. Therefore $\left(T^{m}\right)^{n} T^{*}=T^{*}\left(T^{m}\right)^{n}$ for all $m \geq 2, n \geq 1$. Consequently, $\left(T^{m}-\lambda\right)^{n} T^{*}=T^{*}\left(T^{m}-\lambda\right)^{n}$ and hence for $x \in H_{0}\left(T^{m}-\lambda\right)$, we have

$$
\begin{aligned}
\lim _{n \rightarrow \infty}\left\|\left(T^{m}-\lambda\right)^{n} T^{*} x\right\|^{\frac{1}{n}} & =\lim _{n \rightarrow \infty}\left\|T^{*}\left(T^{m}-\lambda\right)^{n} x\right\|^{\frac{1}{n}} \\
& \leq \lim _{n \rightarrow \infty}\left\|T^{*}\right\|^{\frac{1}{n}} \lim _{n \rightarrow \infty}\left\|\left(T^{m}-\lambda\right)^{n} x\right\|^{\frac{1}{n}} \\
& =0 .
\end{aligned}
$$

Thus $T^{*} x \in H_{0}\left(T^{m}-\lambda\right)$. That $T x \in H_{0}\left(T^{m}-\lambda\right)$ is obvious.

(ii) It is well known that for a totally paranormal operator $T, H_{0}(T-\lambda)=\operatorname{ker}(T-\lambda)$ for all $\lambda \in \mathbb{C}$ [2]. The class of totally paranormal operators includes the class of hyponormal operators and hence normal operators. Since $T^{m}$ is normal for all $m \geq 2$, we have

$$
H_{0}\left(T^{m}-\lambda\right)=\operatorname{ker}\left(T^{m}-\lambda\right)=\operatorname{ker}\left(T^{m}-\lambda\right)^{*} .
$$

For $\lambda=0, H_{0}\left(T^{m}\right)=\operatorname{ker}\left(T^{m}\right)=\operatorname{ker}\left(T^{* m}\right)$.

(iii) By Remark 2.22, $T_{1}^{m}=\left.T^{m}\right|_{M}$ is hyponormal and hence $H_{0}\left(T_{1}^{m}-\lambda\right)=\operatorname{ker}\left(T_{1}^{m}-\lambda\right) \subseteq$ $\operatorname{ker}\left(T_{1}^{m}-\lambda\right)^{*}$.

(iv) Let $x \in H_{0}(T-\lambda)$ then

$$
\lim _{n \rightarrow \infty}\left\|(T-\lambda)^{n} x\right\|^{\frac{1}{n}}=0
$$

Since $T^{m}-\lambda^{m}=(T-\lambda)\left(T^{m-1}+\lambda T^{m-2}+\cdots+\lambda^{m-2} T+\lambda^{m-1}\right)=(T-\lambda) S$, where $S=\left(T^{m-1}+\right.$ $\left.\lambda T^{m-2}+\cdots+\lambda^{m-2} T+\lambda^{m-1}\right)$, we have,

$$
\begin{aligned}
\lim _{n \rightarrow \infty}\left\|\left(T^{m}-\lambda^{m}\right)^{n} x\right\|^{\frac{1}{n}} & =\lim _{n \rightarrow \infty}\left\|(T-\lambda)^{n} S^{n} x\right\|^{\frac{1}{n}} \\
& \leq \lim _{n \rightarrow \infty}\left\|S^{n}\right\|^{\frac{1}{n}} \lim _{n \rightarrow \infty}\left\|(T-\lambda)^{n} x\right\|^{\frac{1}{n}} \\
& \leq\|S\| \lim _{n \rightarrow \infty}\left\|(T-\lambda)^{n} x\right\|^{\frac{1}{n}} \\
& =0 .
\end{aligned}
$$

Therefore $x \in H_{0}\left(T^{m}-\lambda^{m}\right)$ and $H_{0}(T-\lambda) \subset H_{0}\left(T^{m}-\lambda^{m}\right)$. 
On the otherhand if $S$ is invertible then $(T-\lambda)=S^{-1}\left(T^{m}-\lambda^{m}\right)$. For $x \in H_{0}\left(T^{m}-\lambda^{m}\right)$, we have,

$$
\begin{aligned}
\lim _{n \rightarrow \infty}\left\|(T-\lambda)^{n} x\right\|^{\frac{1}{n}} & =\lim _{n \rightarrow \infty}\left\|S^{-n}\left(T^{m}-\lambda^{m}\right)^{n} x\right\|^{\frac{1}{n}} \\
& \leq\left\|S^{-1}\right\| \lim _{n \rightarrow \infty}\left\|\left(T^{m}-\lambda^{m}\right)^{n} x\right\|^{\frac{1}{n}} \\
& =0 .
\end{aligned}
$$

Consequently, $H_{0}\left(T^{m}-\lambda^{m}\right)=H_{0}(T-\lambda)$ for all $m \geq 2$.

(v) $H_{0}(T-\lambda) \subset \operatorname{ker}\left(T^{m}-\lambda^{m}\right)$ follows from (ii) and (iv).

That $S$ is invertible yields $\operatorname{ker}\left(T^{m}-\lambda^{m}\right)=\operatorname{ker}(T-\lambda)$. Again by (ii) and (iv) $H_{0}(T-\lambda)=$ $\operatorname{ker}(T-\lambda)$.

In general $T \in[n N]$ is not translation invariant.

Example 2.24. It is easily seen that, for $T=\left(\begin{array}{cc}1 & 1 \\ -1 & 0\end{array}\right) \in[3 N]$,

$$
\begin{aligned}
& (T-i)^{3}(T-i)^{*}=\left(\begin{array}{cc}
-6 i-8 & -4 i+7 \\
10 i-1 & -4 i-7
\end{array}\right) . \\
& (T-i)^{*}(T-i)^{3}=\left(\begin{array}{cc}
-6 i-8 & -10 i+1 \\
4 i-7 & -4 i-7
\end{array}\right) .
\end{aligned}
$$

Therefore $(T-i) \notin[3 N]$. Thus $T \in[n N]$ is not translation invariant.

Naturally in view of the above statement, the following question arises: What could be the nature of class $[n N]$ operators satisfying the translation invariant property?

In [8] Eungil Ko proved that if the $k^{t h}$ root of a hyponormal operator is translation invariant then it is hyponormal. We use the same technique to prove the following Theorem.

Theorem 2.25. Suppose $T \in[n N]$ is translation invariant then $T$ is normal.

\section{Proof.}

$$
\begin{aligned}
(T-\lambda)^{n}(T-\lambda)^{*} & =(T-\lambda)^{*}(T-\lambda)^{n} \\
0 & =(T-\lambda)^{*}(T-\lambda)^{n}-(T-\lambda)^{n}(T-\lambda)^{*} \\
0 & =(T-\lambda)^{*}\left[\sum_{k=0}^{n}\left(\begin{array}{l}
n \\
k
\end{array}\right) T^{n-k}(-\lambda)^{k}\right]-\left[\sum_{k=0}^{n}\left(\begin{array}{l}
n \\
k
\end{array}\right) T^{n-k}(-\lambda)^{k}\right](T-\lambda)^{*}
\end{aligned}
$$

Put $\lambda=\rho e^{i \theta}, \rho>0,0 \leq \theta<2 \pi$, in (2.4) and dividing the simplified equation by $\rho^{n-1}$ gives,

$$
0=n\left(T^{*} T-T T^{*}\right) e^{(n-1) i \theta}+\frac{1}{\rho}(\text { the other terms }) .
$$

Taking limit as $\rho \rightarrow \infty$ gives, $T T^{*}=T^{*} T$. 


\section{Acknowledgement}

The authors would like to thank the referee for the valuable comments which helped us to improve this manuscript.

\section{References}

[1] P. Aiena, Fredholm and Local Spectral Theory, with Applications to Multipliers, Kluwer Academic Publishers, Dordrecht, Boston, London, 2004.

[2] P. Aiena, M. T. Biondi and F. Villafañe, Weyl's theorems and Kato spectrum, Divulgaciones Matemáticas, 15(2007), 123-142.

[3] T. Ando, Operators with a norm condition, Acta Sci. Math. Szeged, 33(1972), 169-178.

[4] S. K. Berberian, Extensions of a theorem of Fuglede and Putnam, Proc. Am. Math. Soc., 71(1978),113-114.

[5] M. Cho and T. Yamazaki, An operator transform from class A to the class of hyponormal operators and its application, Integral Equ. Oper. Theory, 53 (2005), 497-508.

[6] B. P. Duggal, Tensor products of operators - strong stability and p - hyponormality, Glasgow Math. J., 42(2000), 371-381.

[7] B. P. Duggal, I. H. Jeon and I. H. Kim, On *-paranormal contractions and properties for *-class A operators , Linear Algebra and its Applications, 436 (2012), 954-962.

[8] Eungil Ko, Properties of a kth root of a hyponormal operator, Bull. Korean Math. Soc., 40(2003), 685-692.

[9] D. R. Farenick and I. H. Kim, Tensor products of quasihyponormal operators, Proc. of Kotac, 4(2002), $113-119$.

[10] B. Fuglede, A commutativity theorem for normal operators, Proc. Nat. Acad. Sci. USA, 36(1950), 35-40.

[11] T. Furuta, An extension of the Fuglede-Putnam theorem to subnormal operators using a Hilbert-Schmidt norm inequality, Proc. Am. Math. Soc., 81(1981), 240-242.

[12] H. Heuser, Functional Analysis, Marcel Dekker, New York, 1982.

[13] Jan Stochel, Seminormality of operators from their tensor product, Proc. Am. Math. Soc., 124(1996), 135-140.

[14] I. H. Jeon and B. P. Duggal, On operators with an absolute value condition, J. Korean Math. Soc., 41(2004), 617-627.

[15] A. A. S. Jibril, On n-Power normal operators, The Arabian Journal for Science and Engineering, 33(2008), 247-251.

[16] H. Jinchuan, On the tensor product of operators, Acta Math. Sinica, 9(1993), 195-202.

[17] K. B. Laursen and M. M. Neumann, An Introduction to Local Spectral Theory, Oxford Science Publications (London Math. Soc. Mono. new series 20), (2000).

[18] B. Magajna, On subnormality of generalized derivations and tensor products, Bull. Austral. Math. Soc., 31(1985), 235-243.

[19] J. S. I. Mary and S. Panayappan, Some properties of class A(k) operators and their hyponormal transforms, Glasgow Math. J., 49 (2007), 133-143.

[20] S. Mecheri, K. Tanahashi and A. Uchiyama, Fuglede-Putnam theorem for p-hyponormal or class Y operators, Bull. Korean Math. Soc., 43(2006), 747-753.

[21] M. Putinar, Hyponormal operators are subscalar, J. Operator Theory, 12 (1984), 385-395.

[22] C. R. Putnam, On normal operators in Hilbert spaces, Am. J. Math., 73(1951), 357-362.

[23] T. Saito , Hyponormal operators and related topics, Lecture Notes in Mathematics No.247 (Springer-Verlag, 1971).

[24] Salah Mecheri , On k-quasi-M-hyponormal operators, Mathematical Inequalities and Applications, 16(2013), 895-902.

[25] P. Saphar , Contribution á l'étude des applications linéaires dans un espace de Banach, Bull. Soc. Math. France, 92(1964), 363-384. 
[26] K. Tanahashi, I. H. Jeon, I. H. Kim and A. Uchiyama, Quasinilpotent part of class A or ( $p$, $k$ )-quasihyponormal operators, Operator Theory: Advances and Applications, 187 (2008), 199-210.

[27] A. Uchiyama and K. Tanahashi , Bishop's property ( $\beta$ ) for paranormal operators, Oper. Matrices, 3(2009), 517524.

[28] P. Vrbová, On local spectral properties of operators in Banach spaces, Czechoslovak Math. J., 23(1973), 483492.

Department Of Mathematics, P.S.G College Of Arts And Science, Coimbatore, Tamilnadu, India 641014.

E-mail: stellairenemary@gmail.com

E-mail: sreevijis@gmail.com 